\title{
TWO DIMENSIONAL VERSUS THREE DIMENSIONAL IMAGING IN ENDODONTICS - AN UPDATED REVIEW
}

\author{
Priyank Sethi1, Ritu Tiwari², Maneesha Das ${ }^{3}$, Mahesh Pratap Singh4, Manish Agarwal5, Alfred Joseph Ravikumar ${ }^{6}$ \\ ${ }^{1}$ PhD Scholar, Faculty of Dental Science, Pacific Academy of Higher Education and Research University, Udaipur, Rajasthan. \\ ${ }^{2}$ Consultant, Department of Maxillofacial Radiologist, JSD TechnoDental Imaging Centre, Bangalore. \\ ${ }^{3}$ Senior Lecturer, Department of Conservative Dentistry and Endodontics, Hi-Tech Dental College and Hospital, Bhubaneswar. \\ ${ }^{4}$ Professor and HOD, Department of Conservative Dentistry and Endodontics, People's College of Dental Sciences and Research Centre. \\ ${ }^{5}$ Professor, Department of Conservative Dentistry and Endodontics, People's College of Dental Sciences and Research Centre, Bhopal. \\ ${ }^{6}$ Consultant, Department of Faciomaxillary Surgery, Thirty-Two Dental Care, Porur, Chennai, Tamilnadu.
}

\begin{abstract}
\section{BACKGROUND}

Radiographic imaging is a preliminary and essential step in the diagnosis, treatment planning and followup of all the cases in endodontics. The interpretation of an image can be influenced by several confounding factors including the regional anatomy and superimposition of both teeth and the surrounding dentoalveolar structures. Due to the complexity of the maxillofacial skeleton, conventional two dimensional (2-D) radiographic images fail to provide accurate information of the particular region of interest. Superimposition of the images, seen in planar periapical radiography, reveal only limited information about a three dimensional (3-D) object. The structures so visualised are also subject to geometric distortion. Hence, recent research has highlighted the need for 3-D imaging modality to overcome the potential drawbacks of conventional radiography. Medical Computed tomography (CT) was one of the earliest three dimensional imaging systems. It has been used in the field of endodontics over the past decade but with limited success. This can be ascribed to high radiation exposure, lengthy scan time and cost factor. The next new phase in im aging modalities came with the advent of cone beam computed tomography (CBCT). Contemporary endodontics emphasise on the significance of CBCT, since it limits the radiation exposure and rapidly provides 3D reconstructed images which have been proven to be accurate in all aspects. This paper reviews the importance of three dimensional CBCT technology over conventional two dimensional imaging system along with its potential drawbacks.
\end{abstract}

\section{KEYWORDS}

Cone-Beam Computed Tomography, Dental Radiography, Endodontics, Oral Diagnosis, Three-Dimensional Imaging.

HOW TO CITE THIS ARTICLE: Sethi P, Tiwari R, Das M, et al. Two dimensional versus three dimensional imaging in endodontics An updated review. J. Evolution Med. Dent. Sci. 2016;5(84):6287-6293, DOI: 10.14260/jemds/2016/1420

\section{BACKGROUND}

Diagnostic imaging is considered as an important adjunct in clinical assessment of the patient. Since time immemorial, radiology has played a critical role in dentistry and now with expanding array of different imaging modalities, it has become an indispensable tool in endodontic assessment as well. The first intraoral radiograph to be used in dentistry was reported by Sir C Edmond Kells. ${ }^{1}$ Thereafter, field of radiology has seen several changes. With improvements in the film technology and reduced exposure times, gradually film based system gave way to advanced sensor based digital systems. Solid state sensors like charge coupled device (CCD), complementary metal oxide semiconductor (CMOS) and photostimulable phosphor plates (PSP) largely replaced the film based conventional imaging.2, 3, 4 Digital systems also provided opportunities for image processing and modification (colour, brightness, contrast) which further assisted the diagnostic process. But the images were still a two dimensional representation of three dimensional anatomy and the need for cross sectional information was still unmet.

Financial or Other, Competing Interest: None.

Submission 15-09-2016, Peer Review 09-10-2016,

Acceptance 14-10-2016, Published 20-10-2016.

Corresponding Author:

Dr. Priyank Sethi,

PhD Scholar, Faculty of Dental Science,

Pacific Academy of Higher Education and

Research University, Pacific Hills,

Udaipur, Rajasthan.

E-mail: priyanksethi1987@gmail.com

DOI: $10.14260 /$ jemds/2016/1420

\section{Need for Three Dimensional Visualisation}

The principle of working of an intraoral radiography system is based on the transmission, attenuation and recording of $\mathrm{x}$-rays on a film or digital receptor. It requires optimised geometric configuration of the x-ray machine, object and sensor to provide an accurate projection. If any one of the component of the imaging system is compromised, the resulting image obtained may have errors in geometry or distortion. The images produced by a conventional periapical radiograph are a two dimensional (2D) representation of a three dimensional (3D) area of interest and possess inherent limitations of magnification, distortion and superimposition. ${ }^{5}$ These constraints paved way for the advanced techniques of cross sectional imaging which revolutionised the concept of diagnosis and treatment planning in dentistry. 6,7

\section{Evolution of Cone Beam Computed Tomography System}

Computed Tomography (CT) was invented in 1972 by British engineer Godfrey Hounsfield of EMI Laboratories, England and by South Africa born physicist Allan Cormack of Tufts University, Massachusetts. In 1990, Tachibana and Matsumoto first reported the application of this technology in endodontics. The dental CT also known as DentaScan (GE Healthcare) was first reported by Schwarz et al. ${ }^{8}$ A CT scan utilises a narrow fan-shaped beam of x-ray radiation and multiple exposures around an object to display 3D images in the form of image slices. It was a revolutionary step in diagnosis and treatment as it helped the clinician to assess the morphologic features, pathology and outcome assessment in all the 3D perspectives. Although they produced precise images compared to 2D radiography, the high radiation dose, 
expensive scanner units and lengthy scanning time of these systems limited their popularity. Also the tomographic data captured in medical CT machine was in the form of anisotropic voxels which made the measurements made in multiple planes inaccurate. Hence, keeping in mind the dictum of 'as low as reasonably achievable' (ALARA), newer three dimensional scanning machines were developed. ${ }^{9}$ In 1998, Mozzo et al introduced a new volumetric CT machine (CBCT) that used the cone beam technology for maxillofacial imaging. The first CBCT unit which was approved by the Food and Drug Administration(FDA) in the United States in March 2001 was known by the name New Tom DVT 9000 (Quantitative Radiology SRL, Verona, Italy). Later, other units such as 3D Accuitomo (J. Morita, Kyoto, Japan), i-CAT (Imaging Sciences Int, Hatfield, PA), CB Mercury (Hitachi Med Corp, Chiba-ken, Japan), Galileos (Sirona Dental Systems LLC, Charlotte, NC), Scanora 3D (Soredex, Milwaukee, WI) and Kodak 9500 (Kodak Dental Systems, Rochester, NY) were the various other FDA approved CBCT units manufactured in succession and used in dental practice. Currently, CBCT is considered as a complementary imaging modality for specific applications rather than a replacement for the conventional imaging system. ${ }^{10,11}$

CBCT or Digital volume tomography (DVT) utilises a cone shaped $\mathrm{x}$-ray beam which is centred on a 2-D detector. It performs a single rotation around the object of interest and then captures a series of 160 to 599 basis images. During the rotational exposure, the $\mathrm{x}$-ray source emits radiation and several sequential planar images of the field of view (FOV) are obtained in a complete or sometimes partial arc pattern. Software programs using sophisticated algorithms including back filtered projection are applied for these image data to generate a 3D volumetric data set. These can be used to provide primary reconstruction images in the axial, sagittal and coronal planes (Fig. 1 and Fig. 2). The unit measurements for 2D imaging are called as pixels. But in CBCT, the captured image consists of voxels (3D representation of pixels). CBCT was found to be better than medical CT since it offered significant scan time reduction, reduced radiation dosage and reduced cost for the patient. It requires lesser electrical energy than the fan-shaped beam technology. In CBCT, the entire data is obtained in a course of a single sweep of the scanner and it captures a cylindrical or spherical volume of data known as field of view. Unlike CT scanners, CBCT voxels are isotropic that is equal in all dimensions. This is useful for recording precise measurements of the area of interest. Subjective image quality of CBCT is also high compared to helical CT for the highest resolution modalities. ${ }^{12,13}$

\section{Types of CBCT}

The most common classification of CBCT is based on the dimensions of their field of view or scan volume. Small volume (Also referred to as focused, limited volume) systems possess a maximum scan volume height of $5 \mathrm{~cm}$. Single arch CBCT scans have a FOV height of 5-7 cm within a single arch; interarch CBCT scans have a FOV height of 7-10 cm; maxillofacial CBCT scans have a FOV height which is in the range from 10 $15 \mathrm{~cm}$; and craniofacial CBCT have a FOV height in excess of 15 $\mathrm{cm}$. If the region of interest encompasses the entire jaw portion or entire viscera-cranium, a larger FOV scanning unit should be used. Thus, they are useful in the diagnosis and treatment planning of orthodontic cases, temporomandibular joint analysis, maxillofacial trauma imaging and pathologies involving the jaws. But if only a small area needs to be imaged involving one or more teeth, a smaller FOV may be used using limited CBCT units. They are mostly appropriate for dentoalveolar imaging and in endodontics. Another difference between limited and full CBCT units is that a voxel unit is generally smaller for the limited CBCT unit (0.1-0.2 mm vs. 0.3$0.4 \mathrm{~mm}$ ), hence it offers higher resolution helpful for endodontic applications. ${ }^{14}$

The other methods of classifying CBCT systems are based on the patient position during the scan (Supine, sitting or standing) or functionality of the systems (Standalone or hybrid multimodal systems). Multimodal units are those units which combine digital panoramic and/or cephalometric radiography with a small to medium FOV CBCT system. The main advantage of combining the functions is that these units reduce the overall office footprint for imaging equipment and are less expensive than standalone units as existing robotic panoramic platforms can be re-engineered using small, costeffective detectors. ${ }^{15}$

\section{Exposure}

The CBCT units are manufactured with fixed exposure settings or can also be manually adjusted in relation to the peak kilovoltage $(\mathrm{kVp})$ and/or milliamperage $(\mathrm{mA})$. The operators who use CBCT units with exposure settings which can be adjusted should realise that these parameters directly influence both image quality and patient radiation dose. Therefore, careful selection is required to fulfil the as low as reasonably achievable (ALARA) or as low as practical (ALAP) principles. ${ }^{16,17}$ The adjustment should be done based on the size of the patient and should be according to the manufacturer's recommendations. While $\mathrm{mA}$ may be increased to compensate for an increase in patient size, the ratio of patient-effective dose increases proportionately in the order of 1:1. Adjustment of $\mathrm{kVp}$ has an even greater effect on dose than does $\mathrm{mA}$, hence reducing $\mathrm{kVp}$ to approximately $20 \%$ decreases the radiation dose by nearly $40 \%$, provided all the other parameters remain the same. ${ }^{18}$

\section{Resolution}

There are two types of image resolution - spatial resolution and contrast resolution. Spatial resolution is the ability to show fine details, such as demonstrating the periodontal ligament space or a narrow root canal. Most of the CBCT devices allow choice of this setting. Depending on the type of CBCT unit, lower resolution may be chosen resulting in reduced patient radiation dose. ${ }^{19}$ Contrast resolution is the ability to discriminate between the different types of tissues with very minor differences in x-ray absorption and the differences are displayed in gray levels. Numerous factors limit the contrast resolution of CBCT which includes noise, the resolving capacity of flat-panel detector, refinement of projection geometry, the display characteristics of the monitor and inherent discriminatory limitations of the human eye. Higher image resolution is obtained by higher radiation dosage which is definitely harmful to the patient. Hence, it is important to reduce the exposure parameters to avoid unnecessary exposure of the patient and the clinician to the harmful radiation. Hence, the clinicians who are operating the CBCT must have a thorough understanding of the operational settings and their effects on the quality of the image and radiation safety. 20,21 


\section{Radiation Dosage}

When x-ray radiation exposures are evaluated, the measure of effective dose (E) or radiation is expressed in Sieverts (Sv). The values of $\mathrm{E}$ are calculated based on the relative tissue compositions within the field of view (FOV) and how sensitive they are to radiation. ${ }^{14}$ International Commission on Radiological Protection (ICRP) in 2007 published new factor values for specific organs and tissues while calculating the effective radiation doses. The effective dose calculation for head region imaging include the skin, bone surface, bone marrow, thyroid, oesophagus, salivary glands, brain and the "other" tissues. The effective dose of CBCT units are comparable to panoramic radiography and few intra-oral radiographs but are much lesser compared to the multislice CT machine (Table 1). ${ }^{22,23}$ The effective dose of CBCT is affected by the FOV size, sensitivity of the digital detector, exposure beam type, electric potential power in the $\mathrm{x}$-ray tube, beam geometry and number of rotations around the object to be imaged. But, it should be remembered that the basic principles of as low as reasonably achievable (ALARA) should always be effectively followed to avoid excess radiation exposure. ${ }^{24,25}$

\section{CBCT Softwares}

In 1982, the first prototype CBCT scanner was developed for angiographic usage. Later in 1990s, a CBCT scanner for dentomaxillofacial use was developed and, since the very first report, use of this technique has become widespread in dentistry. ${ }^{26}$ There are a number of softwares available with each type of CBCT unit. These are Sidexis 4, CS 3D, Planmeca Romexis 3D, Sicat Suite, i-Dixel 3D imaging software They give high resolution volumetric image from a small, medium or large FOV to provide an immediate overview of the anatomy such as maxillary sinus cavity, inferior alveolar nerve and the thickness of bone prior to detection of any pathology or implant placement. The images can be instantly viewed from different projections or converted into numerous crosssectional slices. Measuring and annotation tools such as mandibular nerve canal tracing assist in safe and accurate treatment planning for implant placement. It also serves as an excellent patient education tool as shown in Fig. 3.

\section{Recent Advancements}

The most recent advancement is the development of $\mathrm{Tx}$ STUDIO 5.4 software inbuilt in i-CAT CBCT technology. With the help of Tx Studio 5.4 3D software, placing and restoring implants, performing guided surgery and treatment of complex endodontic cases have been extremely easy (Fig. 4 and 5). The rich visual images help in educating and motivating the patient to accept the proposed treatment plan. A significantly more efficient and productive office with the fastest 3D radiographic workflow is available and chair side case work-ups can be completed in minutes with greater precision and lesser radiation dosage ${ }^{27}$ (Fig. 4 and Fig. 5).

\section{Advantages of 3D Imaging over 2D Imaging}

The introduction of CBCT technology in clinical dental practice has provided many advantages for maxillofacial imaging. They are as follows:

\section{Analysis of Root Canal Morphology}

CBCT was found to be a very reliable and non-invasive method to view root canal anatomy in all the spatial planes (Axial, coronal and sagittal) and eliminates the risk of image distortion and anatomic noise .28The accuracy of CBCT and other imaging modalities in the identification of the root canal morphology has been evaluated and compared to the modified canal staining and clearing technique by Neelakantan et al, CBCT was found to correctly identify all the root canals in almost $99.71 \%$ of the cases. It could correctly identify the presence of second MB2 canal in all cases in maxillary molars which were commonly missed out by periapical radiographs. It was found by various studies that the CBCT detection rates increased from $60 \%$ to $93.3 \%$ with increasing resolution suggesting that if $\mathrm{CBCT}$ has to be used, then resolutions in the order of $0.12 \mathrm{~mm}$ or less are optimal.29,30 CBCT can be used along with dental operating microscope for identification of the internal anatomy of maxillary first molar. 31 They can also be used to assess teeth with unusual morphology such as " $\mathrm{C}$ " shaped canals, dilacerated teeth, dens in dente fused teeth or teeth with unusual number of roots. ${ }^{32,33,34}$

2. Assessment of Root Canal Preparations and IntraOperative Procedures

Periapical radiographs give only two dimensional limited information about a three dimensional root canal system. CBCT has been very effective non-invasive method for the measurement of root dentin thickness, canal curvature, canal centre by providing images in orthogonal as well as oblique planes. Thus, CBCT images permit nondestructive and metrically exact analysis of variables such as volume, surface area, cross sectional shape and taper of the canal. They are also useful during intra-operative procedures such as detecting missed canals during retreatment, broken instruments, perforations and calcified canals. 35,36

\section{Detection of Periradicular Lesions}

For a lesion to be visible on a periapical radiography, minimum of $30-50 \%$ of bone loss should be present. Lesions within the cancellous bone generally cannot be detected by conventional 2D radiography unless there is extensive destruction of the bone cortex on the outer surface, or there is erosion of the cortical bone from the inner surface. CBCT can reveal bone defects present in the cancellous and cortical bone separately even at the earliest stages of the pathological event. This was mostly because there was no superimposition of the cortical bone over the lesion by elimination of anatomic noise. ${ }^{37}$ Estrela et $\mathrm{al}^{38}$ demonstrated the higher prevalence of apical periodontitis diagnosed by CBCT imaging compared to conventional radiography. Thus, early diagnosis of periradicular radiolucent changes by CBCT permitted the clinician to early identify and modify their treatment plan thereby giving a positive treatment outcome. In asymptomatic apical periodontitis, CBCT was much more sensitive in detecting the existing periapical radiolucency which routine radiographic examinations failed to diagnose. ${ }^{39}$ They were also useful in detection of lesions close to the maxillary sinus, sinus membrane thickening and when there was less than $1 \mathrm{~mm}$ bone present between the lesion and floor of maxillary sinus in cases of posterior teeth. 40 CBCT was also useful in cases of diagnostic dilemma where benign and malignant lesions 
like carcinoma and odontogenic cyst mimicked periapical lesions when viewed by radiographs alone. Thus, it provides the clinician with great detail and much information and proving the presence of any previously undiagnosed pathosis or any odontogenic aetiology of pain. ${ }^{41}$

\section{Diagnosis of Traumatic Injuries}

The diagnosis of root fractures and cortical bone fractures after traumatic injuries are based on clinical findings, sensitivity tests and radiographic examination. Horizontal root fractures (HRF) are generally detected taking multiple angled periapical radiographs. However, they might not still be properly visualised in the radiographs. 3D CBCT is useful by providing high resolution images in the 3 planes with no errors in geometry of the image. 42 Vertical root fractures (VRF) or longitudinal root fractures are difficult to diagnose since the clinical signs and symptoms are nonspecific and can often be overlooked if the incident $x$-ray beam is not parallel to the plane of fracture line while taking periapical radiographs. CBCT reconstructed data provide precise information to clearly visualise the fracture line in the axial, coronal and sagittal plane with just a single exposure. It is advantageous over medical CT since images are produced at a faster speed and lower doses of radiation. ${ }^{43}$ Edlund et $\mathrm{al}^{44}$ evaluated the diagnostic efficacy of CBCT in suspected vertical root fractures in endodontically treated teeth using exploratory surgery and found that the positive predictive value of CBCT was 91\% making it the most reliable tool in the diagnosis of VRF. Generally, CBCTs with a smaller field of view are more sensitive in detecting VRF than larger field of view systems. 45

\section{Diagnosis of Root Resorption and Perforations}

Conventional periapical radiographs provide limited clinical information with respect to the three dimensional defect such as root resorption. It is unable to reveal the exact location and nature of the resorptive defect or thickening of remaining root canal dentine particularly in the bucco-lingual direction. There is further image distortion and superimposition of various anatomic structures resulting in limited diagnostic information. 3D CBCT reconstructed images are useful in the diagnosis of the size of the defect as well as its proximity to the root canals. ${ }^{46,47}$ The CBCT voxels being isotropic ensures that the images produced are completely accurate geometrically and free from distortion, thus accurately differentiating between external and internal type of root resorption.48 Additionally, they are also useful in identifying the portal of entry of the periapical lesions to differentiate invasive cervical resorption from internal root resorption. CBCT imaging also allows for better visualisation of the perforation site in various sections and angulations without any geometric distortion of images. 49

6. Assessment of the Quality of Root Canal Treatment and Outcome Assessment

CBCT is used as a superior imaging modality in assessment of teeth with ideal root canal treatment and in cases where canals are filled short of the apex. CBCT was useful for accurately measuring the working length of teeth (Precision varied between 0.41-0.51 $\mathrm{mm}$ ) when compared with the electronic apex locators which are considered as gold standard method for working length estimation. It was useful in patients with cardiac pacemakers where the use of an apex locator is contraindicated and periapical radiography is unreliable.50,51 Periapical radiography can't detect accurately if there is any existing periapical lesions post endodontic treatment. But, CBCT was useful in detecting the asymptomatic non-healed lesions during the posttreatment followup period to identify the cases requiring retreatment. 52

\section{Pre-surgical Assessment}

Conventional radiography gives limited information in the buccal-lingual plane and the presence of the buccal plate interferes while estimating the defects in osseous structures such as periapical lesions. Distortion of images in panoramic radiographs has also been well documented and quantified, making it a challenging medium to gain accurate measurements. Use of CBCT has been recommended for endodontic surgery treatment planning since it enables the assessment of the lesion in three dimensional plane in terms of its location, extent, position of the roots within the bone, proximity to anatomic vital structures such as inferior alveolar nerve, mental foramen, maxillary sinus and nasal cavity. ${ }^{53}$ It also allows better visualisation of the extruded endodontic material and its proximity to the inferior alveolar nerve and mental foramen than 2D radiography. ${ }^{54}$ Periapical radiographs are found to be less sensitive in detecting lesions associated with the upper molar teeth since the root apices are in very close proximity to the floor of the maxillary sinus (Anatomic noise). CBCT is very effective in such cases by providing 3D image without superimposition. ${ }^{40}$ The role of CBCT in providing accurate linear measurements and 3D evaluation of the alveolar ridge with fabrication of the surgical guides is also useful during placement of implants. ${ }^{55}$

\section{Disadvantages of CBCT}

CBCT images also possess certain limitations like formation of image artefacts, graininess of the image and poor soft tissue contrast. CBCT image artefacts are mostly due to the following possible sources: The patient, the scanner, x-ray beam artefacts and artefacts related to the CBCT system like partial volume averaging, under sampling and cone beam effect. The cone beam effect occurs mostly in the peripheral portions of the scan volume mostly due to the divergence of X-ray beam. As a result, less information is obtained for the peripheral structures resulting in streaking artefacts, image distortion and greater peripheral noise. Image artefacts can also occur due to the inherent polychromatic nature of $x$-ray beam which is known as beam hardening. Two types of artefacts are observed due to beam hardening. They are (1) Cupping artefact is the distortion of metallic structures due to the differential absorption of x-ray beam and (2) streaks and dark bands which can be present between two dense objects. ${ }^{56}$ Graininess of the image might be due to remaining noise occurring in the CBCT systems with large FOV mostly when a low signal is used to attempt to reduce the radiation exposure. 
Such artefacts can be eliminated by reducing the FOV to avoid scanning structures outside the ROI which are susceptible to beam hardening. Poor soft tissue contrast is also seen in CBCT due to many factors which includes the $\mathrm{x}$-ray beam being divergent in nature causing large variation or non-uniformity of the x-ray beam incident on the patient and non-uniform absorption with greater signal-to-noise ratio on the cathode aspect of image relative to the anode side. Also, the flat panel detector based artefacts can affect its response to the x-ray radiation. Recent scientific reports have discussed about the increasing professional concerns over the potential association between radiation exposure and cancer. ${ }^{57,58}$

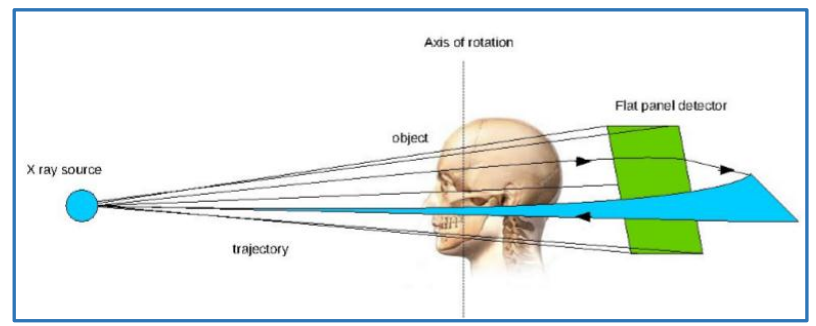

Figure 1. Schematic Representation of the working Mechanism of a CBCT Scan unit

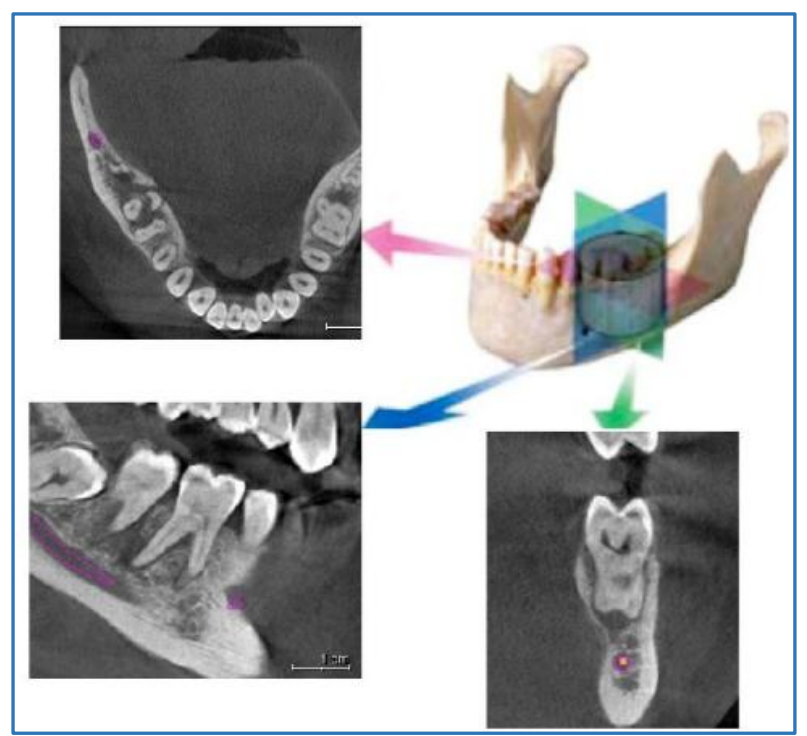

Figure 2. Standard Display modes of CBCT Volumetric data in the Axial, Sagittal and Coronal Plane

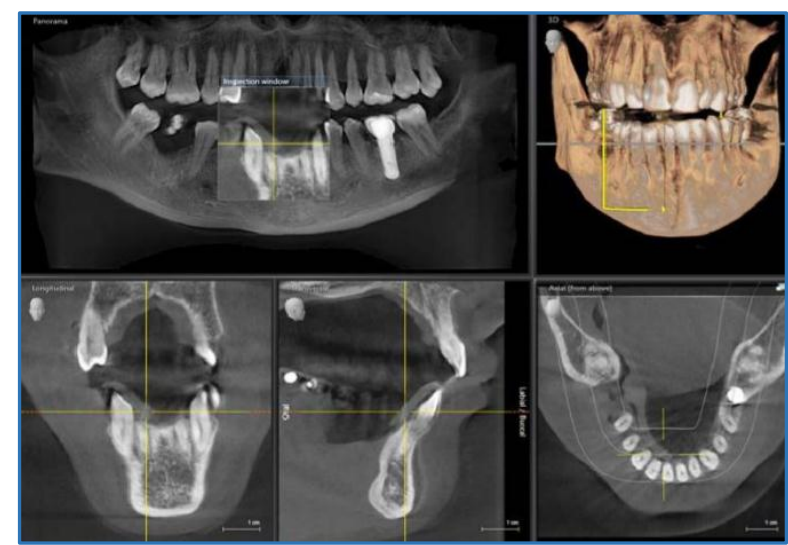

Figure 3. Targeted Display and Editing of a 3D data Volume and its Cross sectional views Visualised by the CBCT software (CS-3D)
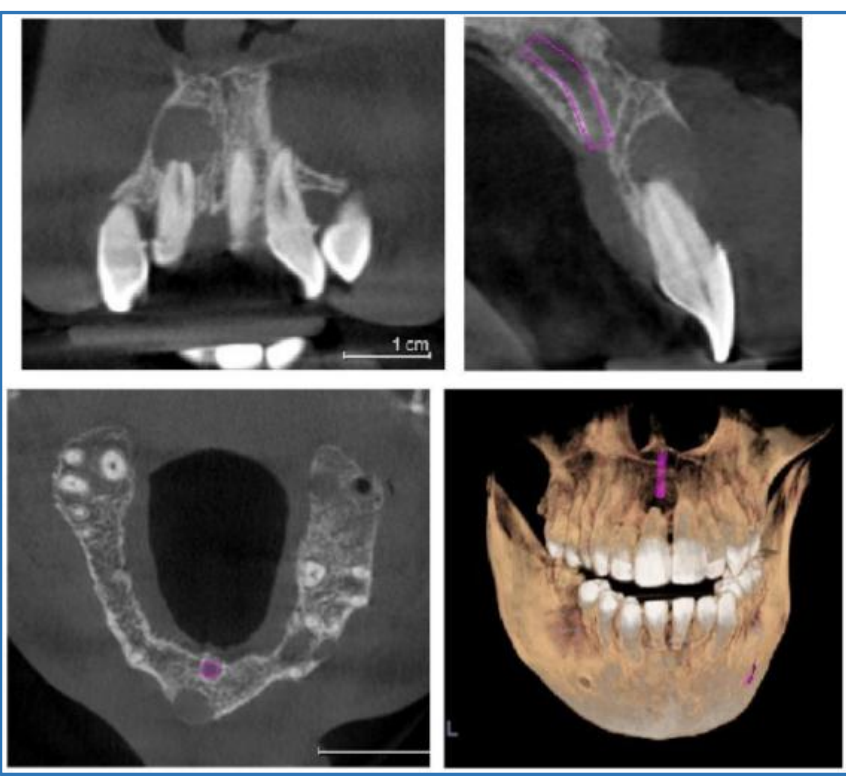

Figure 4. Evaluation of Bone Density in Various Planes of view before Planning for Implant Placement using Tx Studio (5.4) Software

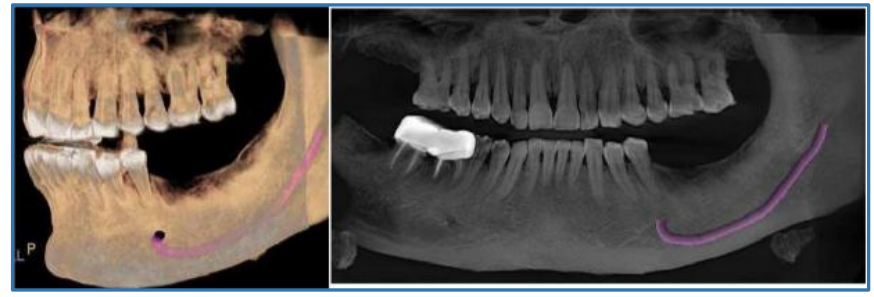

Figure 5. 3D Views to Visualise the Inferior Alveolar Nerve Position during Pre-surgical planning for Placement of Implant by CS-3D Software

\begin{tabular}{|lc|}
\hline Imaging modality & $\begin{array}{l}\text { Effective dose } \\
(\mu \mathrm{Sv})\end{array}$ \\
Intra-oral (film or digital ) radiograph -(1) & $<8.3$ \\
Dental bite-wing radiographs (F-speed)- (4) & 38 \\
Full mouth series radiographs & $35-388$ \\
Panoramic radiograph & $9-26$ \\
Cephalometric radiograph & $2-6$ \\
Cone beam CT (Dentoalveolar-Small and Medium & $4.7-38.3$ \\
FOV) & \\
Cone beam CT ( Craniofacial-Large FOV) & $68-1073$ \\
Medical CT (Head) & 2000 \\
\hline
\end{tabular}

Table 1. Comparison of Effective Radiation Dosage among the Various Imaging Modalities

\section{CONCLUSION}

The advent of the three dimensional CBCT imaging system has provided the clinician a powerful tool to facilitate interactive image manipulation and enhancement, thus significantly increasing the amount of information gleaned from a volume. This relatively modern state-of-art imaging technology has added another dimension to dental radiography and is quickly becoming the gold standard for radiographic imaging in dentistry. Maxillofacial CBCT imaging provides very accurate, submillimetre resolution images of great diagnostic quality, enabling 3D visualisation of the complex osseous structures of 
the maxillofacial region. In clinical endodontics, the application of CBCT should be based on a benefit-risk analysis.

Since CBCT utilises ionising radiation, patient exposure should be kept as low as reasonably achievable (ALARA) to avoid unnecessary radiation hazards. Operators of the CBCT equipment should be aware of the effects of additional exposure settings on both the image quality and patient radiation dose. Hence, oral health professionals have an ethical responsibility to become familiar with the technical and operational aspects of CBCT, as well as understand the scientific validity and associated health risks of its applications.

\section{REFERENCES}

1. Jacobsohn PH, Fedran RJ. Making darkness visible: the discovery of x-ray and its introduction to dentistry. J Am Dent Assoc 1995;126(10):1359-67.

2. Langland OE, Langlais RP. Early pioneers of oral and maxillofacial radiology. Oral Surg Oral Med Oral Pathol Oral Radiol Endod 1995;80(5):496-511.

3. Todd R. Dental imaging-2D to 3D: a historic, current and future view of projection radiography. Endod Topics 2014;31(1):36-52.

4. Van der Stelt PF. Filmless imaging: the uses of digital radiography in dental practice. J Am Dent Assoc 2005;136(10):1379-87.

5. American dental association council on scientific affairs. The use of dental radiographs: update and recommendations. J Am Dent Assoc 2006;137(9): 1304-12.

6. Scarfe WC. Imaging of maxillofacial trauma: evolutions and emerging revolutions. Oral Surg Oral Med Oral Pathol Oral Radiol Endod 2005;100(2 Suppl):S75-96.

7. Danforth RA, Dus I, Mah J. 3-D volume imaging for dentistry: a new dimension. J Calif Dent Assoc 2003;31(11):817-23.

8. Schwarz MS, Rothman SL, Rhodes ML, et al. Computed tomography: part I. preoperative assessment of the mandible for endosseous implant surgery. Int J Oral Maxillofac Implants 1987;2(3):137-41.

9. Farman AG, Levato CM, Scarfe WC. 3D X-ray: an update. Inside Dentistry 2007;3(6):70-4.

10. Mozzo P, Procacci C, Tacconi A, et al. A new volumetric CT machine for dental imaging based on the cone beam technique: preliminary results. Eur Radiol 1998;8(9):1558-64.

11. White SC, Pharoah MJ. The evolution and application of dental maxillofacial imaging modalities. Dent Clin N Am 2008;52(4):689-705.

12. Scarfe WC, Farman AG. What is cone-beam CT and how does it work? Dent Clin North Am 2008;52(4):707-30.

13. Hashimoto K, Kawashima S, Araki M, et al. Comparison of the image performance between cone-beam computed tomography for dental use and four row multidetector helical CT. J Oral Sci 2006;48(1):27-34.

14. Scarfe WC, Levin MD, Gane D, et al. Use of cone beam computed tomography in endodontics. Int J Dent 2009;2009:1-20.

15. Scarfe WC, Li Z, Aboelmaaty W, et al. Maxillofacial cone beam computed tomography: essence, elements and steps to interpretation. Aust Dent J 2012;57(Suppl 1): 46-60.
16. National Council on Radiation Protection \& Measurements. NCRP Report No: 145, Radiation protection in dentistry. Available at: ncrponline.org/Publications/Press_Releases/145press.html. Accessed March 18, 2014.

17. Farman AG. ALARA still applies. Oral Surg Oral Med Oral Pathol Oral Radiol Endod 2005;100(4):395-7.

18. Palomo JM, Rao PS, Hans MG. Influence of CBCT exposure conditions on radiation dose. Oral Surg Oral Med Oral Pathol Oral Radiol Endod 2008;105(6):773-82.

19. Kwong JC, Palomo JM, Landers MA, et al. Image quality produced by different CBCT settings. Am J Orthod Dentofac Orthoped 2008;133(2):317-27.

20. Tsiklakis K, Donta C, Gavala S, et al. Dose reduction in maxillofacial imaging using low dose cone beam CT. Eur J Radiol 2005;56(3):413-7.

21. Qu XM, Li G, Ludlow JB, et al. Effective radiation dose of ProMax 3D cone-beam computerized tomography scanner with different dental protocols. Oral Surg Oral Med Oral Pathol Oral Radiol Endod 2010;110(6):770-6.

22. Valentin J. The 2007 recommendations of the international commission on radiological protection. Publication 103. Annals of the ICRP 2009;37:2-4.

23. White SC, Pharoah MJ. Oral radiology: principles and interpretation. Mosby Elsevier, St. Louis, Missouri 2009.

24. Ludlow JB, Ludlow LED, Brooks SL, et al. Dosimetry of 3 CBCT devices for oral and maxillofacial radiology: $\mathrm{CB}$ mercuray, new tom 3G and i-CAT. Dentomaxillofac Rad 2006;35(4):219-26.

25. Ludlow JB, Ivanovic M. Comparative dosimetry of dental CBCT devices and 64-slice CT for oral and maxillofacial radiology. Oral Surg Oral Med Oral Pathol Oral Radiol Endod 2008;106(1):106-14.

26. Abella F, Morales K, Garrido I, et al. Endodontic applications of cone beam computed tomography: case series and literature review. Giornale Italiano di Endodonzia 2015;29(2):38-50.

27. Weber MT, Stratz N, Fleiner J, et al. Possibilities and limits of imaging endodontic structures with CBCT. Swiss Dent J 2015;125(3):293-311.

28. Michetti J, Maret D, Mallet JP, et al. Validation of cone beam computed tomography as a tool to explore root canal anatomy. J Endod 2010;36(7):1187-90.

29. Neelakantan P, Subbarao C, Subbarao CV. Comparative evaluation of modified canal staining and clearing technique, cone-beam computed tomography, peripheral quantitative computed tomography, spiral computed tomography and plain and contrast medium enhanced digital radiography in studying root canal morphology. J Endod 2010;36(9):1547-51.

30. Bauman R, Scarfe W, Clark S, et al. Ex vivo detection of mesiobuccal canals in maxillary molars using CBCT at four different isotropic voxel dimensions. Int Endod J 2011;44(8):752-8.

31. Filho FB, Zaitter S, Haragushiku GA, et al. Analysis of the internal anatomy of maxillary first molars by using different methods. J Endod 2009;35(3):337-42.

32. Zheng Q, Zhang L, Zhou X, et al. C-shaped root canal system in mandibular second molars in a Chinese population evaluated by cone-beam computed tomography. Int Endod 2011;44(9):857-62. 
33. Kaneko T, Sakaue H, Okiji T, et al. Clinical management of dens invaginatus in a maxillary lateral incisor with the aid of cone beam computed tomography - a case report. Dent Traumatol 2011;27(6):478-83.

34. Abella F, Mercadé M, Sindreu FD, et al. Managing severe curvature of radix entomolaris: three-dimensional analysis with cone beam computed tomography. Int Endod J 2011;44(9):876-85.

35. Estrela C, Bueno MR, Sousa-Neto MD, et al. Method for determination of root curvature radius using cone-beam computed tomography images. Braz Dent J 2008; 19(2): 114-8.

36. Randy LB, Barbizam JV, Cohenca N. Intraoperative endodontic applications of cone-beam computed tomography. J Endod 2013;39(4):548-57.

37. Liang $\mathrm{YH}$, Jiang $\mathrm{L}$, Gao $\mathrm{XJ}$, et al. Detection and measurement of artificial periapical lesions by cone-beam computed tomography. Int Endod J 2014;47(4):332-8.

38. Estrela C, Bueno MR, Leles CR, et al. Accuracy of cone beam computed tomography and panoramic and periapical radiography for detection of apical periodontitis. J Endod 2008;34(3):273-9.

39. Paula-Silva FWG, Wu MK, Leonardo MR, et al. Accuracy of periapical radiography and cone beam computed tomography scans in diagnosing apical periodontitis using histological findings as a gold standard. J Endod 2009;35(7):1009-12.

40. Low MTL, Dula KD, Burgin W, et al. Comparison of periapical radiography and limited cone-beam tomography in posterior maxillary teeth referred for apical surgery. J Endod 2008;34(5):557-62.

41. Sekerci AE, Sisman Y, Etoz M, et al. Aberrant anatomical variation of maxillary sinus mimicking periapical cyst: a report of two cases and role of CBCT in diagnosis. Case Rep Dent 2013;2013:1-4.

42. Kamburoglu K, Cebeci ARI, Grondahl HG. Effectiveness of limited cone-beam computed tomography in the detection of horizontal root fracture. Dental Traumatol 2009;25(3):256-61.

43. Wang P, Yan XB, Lui DG, et al. Detection of dental root fractures by using cone-beam computed tomography. Dentomaxillofac Radiol 2011;40(5):290-8.

44. Edlund M, Nair MK, Nair UP. Detection of vertical root fractures by using cone-beam computed tomography: a clinical study. J Endod 2011;37(6):768-72.

45. Bernardes RA, Moraes de IG, Hungaro MA, et al. Use of cone-beam volumetric tomography in the diagnosis of root fractures. Oral Surg Oral Med Oral Pathol Oral Radiol Endod 2009;108(2):270-7.
46. Cohenca N, Simon JH, Mathur A, et al. Clinical indications for digital imaging in dentoalveolar trauma. Part 2: root resorption. Dental Traumatol 2007;23(2):105-13.

47. Patel S, Dawood A, Wilson R, et al. The detection and management of root resorption lesions using intraoral radiography and cone beam computed tomography - an in vivo investigation. Int Endod J 2009;42(9):831-8.

48. D'Addazio PSS, Campos CN, Ozcan M, et al. A comparative study between cone-beam computed tomography and periapical radiographs in the diagnosis of simulated endodontic complications. Int Endod J 2010;44(3): 218-24.

49. Estevez R, Aranguren J, Escorial A, et al. Invasive cervical resorption class III in a maxillary central incisor: diagnosis and follow-up by means of cone-beam computed tomography. J Endod 2010;36(12):2012-4.

50. Demiralp KO, Kamburoglu K, Gungor K, et al. Assessment of endodontically treated teeth by using different radiographic methods: an ex vivo comparison between CBCT and other radiographic techniques. Imaging Sci Dent 2012;42(3):129-37.

51. Connert T, Hulber MJ, Godt A, et al. Accuracy of endodontic working length determination using cone beam computed tomography. Int Endod J 2014;47(7):698-703.

52. Liang YH, Li G, Wesselink PR, et al. Endodontic outcome predictors identified with periapical radiographs and cone-beam computed tomography scans. J Endod 2011;37(3):326-31.

53. Bornstein MM, Lauber R, Sendi P, et al. Comparison of periapical radiography and limited cone-beam computed tomography in mandibular molars for analysis of anatomical landmarks before apical surgery. J Endod 2011;37(2):151-7.

54. Moura MS, Guedes OA, Goncalves AH, et al. Influence of length of root canal obturation on apical periodontitis detected by periapical radiography and cone beam computed tomography. J Endod 2009;35(6):805-9.

55. Guerrero ME, Jacobs R, Loubele M, et al. State-of-the-art on cone beam CT imaging for preoperative planning of implant placement. Clin Oral Invest 2006;10(1):1-7.

56. Schulze R, Heil U, Grob D, et al. Artifacts in CBCT: a review. Dent Maxillofac Radiol 2011;40(5):265-73.

57. Katsumata A, Hirukawa A, Noujeim $M$, et al. Image artifacts in dental cone beam CT. Oral Surg Oral Med Oral Pathol Oral Radiol Endod 2006;101(5):652-7.

58. Claus EB, Calvocoressi L, Bondy ML, et al. Dental x-rays and risk of meningioma. Cancer 2012;118(18):4530-7. 